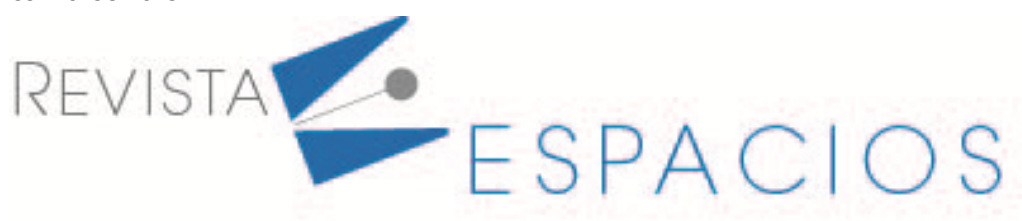

\title{
¿Cómo conseguir legitimidad? Una revisión sistemática
}

\author{
How to achieve legitimacy? A systematic review
}

\author{
GORDO MOLINA, Virginia ${ }^{1}$ \\ DIEZ MARTIN, Francisco ${ }^{2}$
}

\begin{abstract}
Resumen
Las empresas necesitan conseguir y mantener legitimidad para acceder a los recursos y aumentar sus probabilidades de supervivencia. Las numerosas investigaciones en este campo, durante las últimas décadas, hace necesario mostrar una visión global del mismo. El propósito de esta investigación es identificar las principales estrategias empresariales para conseguir legitimidad. Se realiza una revisión sistemática de investigaciones sobre legitimidad organizacional siguiendo la guía PRISMA. Este trabajo identifica la relación entre las estrategias y el tipo de legitimidad asociada.

Palabras clave: legitimidad, estrategia, emprendimiento, empresas.
\end{abstract}

\begin{abstract}
Companies need to gain and maintain legitimacy to access resources and increase their chances of survival. The numerous investigations in this field, during the last decades, make it necessary to show a global vision of it. The purpose of this research is to identify the main business strategies to achieve legitimacy. A systematic review of research on organizational legitimacy is carried out following the PRISMA guide. This work identifies the relationship between strategies and the type of associated legitimacy.

key words: legitimacy, Strategy, Entrepreneurship, Firms
\end{abstract}

\section{Introducción}

La legitimidad ha recibido una atención importante por parte de los investigadores en teoría institucional (Meyer \& Rowan, 1977; Oliver, 1991; Suchman, 1995) por ser un elemento crucial para la supervivencia y crecimiento de la empresa. En particular, cómo obtiene una empresa la legitimidad ha sido objeto de numerosas investigaciones, cobrando importancia tanto para los empresarios como entre los académicos (e.g. Deephouse \& Suchman, 2008; Delmar \& Shane, 2004; Zimmerman \& Zeitz, 2002).

"Organizational legitimacy is the perceived appropriateness of an organization to a social system in terms of rules, values, norms, and definitions" (Deephouse et al., 2017, p.32). La legitimidad es un recurso importante para adquirir otros recursos que son cruciales para el crecimiento de nuevas empresas y la legitimidad puede ser mejorada por acciones estratégicas (Zimmerman \& Zeitz, 2002). Una nueva empresa puede obtener un mayor rendimiento económico si se legitima satisfaciendo las expectativas cognitivas, regulativas y normativas de los stakeholders (Wang et al., 2017).

\footnotetext{
${ }^{1}$ Doctoranda. Universidad Rey Juan Carlos. Docente. RCU. Escorial - María Cristina. virginiagm2017@gmail.com

2 Director del Master Universitario en Alta Dirección. Departamento de Economía de la Empresa. Universidad Rey Juan Carlos. francisco.diez@urjc.es
} 
Si una empresa no se considera legitima por los grupos de interés tendrá dificultades para atraer recursos necesarios, inversores, clientes y poder desarrollar su actividad. Para ello, la empresa debe alcanzar el umbral de legitimidad (McKnight \& Zietsma, 2018; Nagy et al., 2017). Pero, no basta con que una empresa sea o no legitima ni que haya un umbral de legitimidad único. Las presiones de legitimidad pueden variar a medida que maduran las empresas (Tracey et al., 2018). Una empresa, a medida que va creciendo o cambiando de etapa en su ciclo de vida organizacional, debe seguir cumpliendo con las expectativas de las audiencias que van cambiando, por ejemplo, integrando su identidad organizativa (Fisher et al., 2016).

A todo esto se añade, que las acciones que pueden realizar las empresas de manera proactiva para lograr alcanzar la legitimidad son diversas: a través del discurso (e.g. Green et al., 2009); a través de acciones simbólicas que aumenten la credibilidad de la empresa (e.g. Zott \& Huy, 2007); a través de los medios de comunicación (e.g. Petkova et al., 2013); mediante la implantación de programas de calidad (e.g. Westphal et al., 1997); mediante estrategias de conformidad, diferenciación o manipulación del entorno (e.g. Zimmerman \& Zeitz, 2002); mediante la adopción de valores sociales (e.g. Chiu \& Sharfman, 2011); mediante la creación de alianzas y asociaciones con otras empresas (e.g. Plummer et al., 2016), entre otras muchas acciones.

La pregunta de investigación de este estudio es conocer qué acciones y estrategias deben realizar las empresas para lograr, mantener o perder la legitimidad y con qué tipo de legitimidad se corresponden. Esta investigación es necesaria principalmente por dos motivos: (1) por el número tan elevado de publicaciones en relación a las estrategias para conseguir legitimidad, lo cual hace que sea difícil un seguimiento de la evolución del campo y hace necesario una revisión de los trabajos de investigación publicados. Por ejemplo, sólo en la base de datos de Web of Science (WOS), entre el período de 1992-2019, se obtienen 14.111 publicaciones con este término y (2) porque no hemos encontrado en las publicaciones científicas un documento que recoja de manera única o resumida nuestro objeto de estudio. Si bien, Suchman (1995) realizó un estudio de las estrategias de legitimidad, pero existen numerosas publicaciones posteriores al trabajo de dicho autor, que hacen necesario actualizar las estrategias no analizadas en dicho estudio y relacionarlas con otros tipos de legitimidad que han aparecido desde entonces.

Para abordar nuestra pregunta de investigación y darla respuesta, el primer objetivo es identificar, organizar e interpretar las investigaciones pasadas y actuales sobre las estrategias o acciones que deben realizar las empresas para lograr, mantener o perder la legitimidad. Para ello, se realizó una revisión sistemática de la literatura sobre las estrategias de legitimidad. Un segundo objetivo de este trabajo es relacionar esas estrategias o acciones con el tipo de legitimidad en que mayormente inciden. Los tipos de legitimidad son muy diversos. Por ejemplo, Suchman (1995) distingue tres tipos de legitimidad: legitimidad pragmática, legitimidad moral y legitimidad cognitiva; Archibald (2004) distingue dos tipos de legitimidad: legitimidad sociopolítica (regulativa) y legitimidad cultural (combinó la legitimidad normativa y cognitiva). Díez-de-Castro et al. (2018) recopilan hasta 8 tipos de legitimidad centrada en la gestión empresarial: legitimidad cognitiva (cultural), legitimidad regulatoria, legitimidad moral (ética), legitimidad pragmática (instrumental o recursos), legitimidad gerencial (producto), legitimidad técnica, legitimidad emocional (relacional) y legitimidad de la industria o del sector.

El documento está organizado de la siguiente manera: en la siguiente sección, se presentan la metodología. A continuación se muestran los resultados del análisis de la revisión sistemática y, por último, incluye las conclusiones, limitaciones del estudio y propuestas para futuras investigaciones.

\section{Metodología}

Para conocer las estrategias o acciones que tienen a su disposición las empresas para lograr alcanzar la legitimidad, mantenerla o perderla se lleva a cabo una revisión sistemática de la literatura. Una revisión sistemática es un método utilizado para identificar las investigaciones relevantes, evaluar la calidad de los 
estudios publicados y realizar un trabajo de síntesis de los hallazgos obtenidos por los estudios publicados (Crowther et al., 2010; Knopf, 2006; V. Smith et al., 2011). Utiliza métodos explícitos, sistemáticos, rigurosos, transparentes para minimizar el sesgo y proporcionar hallazgos y pruebas confiables (Cook et al., 1997). Por tanto, en una revisión sistemática se especifica con detalle el proceso de elaboración. Se pueden realizar revisiones sistemáticas utilizando un análisis narrativo o un meta-análisis. Existen distintos tipos de revisiones: Systematic review; Meta-analysis; Qualitative synthesis; Mixed studies review; Integrative review; Scoping review; RE-AIM review; Umbrella review (Whittemore et al., 2014).

Para que todos los informes en las revisiones sistemáticas contengan unas pautas estándares y sigan unos protocolos, que conlleve a mejorar la transparencia de los informes de las revisiones sistemáticas y mejorar la coherencia en las revisiones sistemáticas, se han recomendado los elementos de informes para las revisiones sistemáticas y meta-análisis PRISMA (Preferred Reporting Items of Systematic reviews and Meta-Analyses). La guía PRISMA consiste en una lista de 27 ítems destinada a facilitar la preparación de una revisión sistemática. El diagrama de PRISMA divide en cuatro fases el proceso de revisión: identificación, cribado, elegibilidad e inclusión (Moher et al., 2009). La fase de identificación consiste en el número de registros identificados a través de la búsqueda en la base de datos y a través de otras fuentes. La fase de cribado consiste en eliminar el número de registros duplicados, así como en determinar que número de registro son seleccionados y cuales excluidos. La fase de elegibilidad o idoneidad consiste en determinar que artículos a texto completo son evaluados y cuales a texto completo son excluidos, especificando los motivos de ello. Por último, en la última fase de inclusión se determina el número de artículos incluidos para la síntesis cualitativa y cuantitativa (meta-análisis).

\subsection{Datos}

En la construcción de la base de datos, para realizar la primera fase de identificación de la guía PRISMA, se seleccionaron artículos de revistas científicas procedentes de la base de datos de Social Science Citation Index (SSCl) y Emerging Sources Citation Index (ESCl) de la Colección Principal de Web of Science (WOS). En la selección de datos se realizaron dos búsquedas. La metodología de realizar dos búsquedas en WOS, filtrar por artículos de revistas y áreas de investigación se tomo siguiendo algo parecido a lo realizado por los autores Urbano et al. (2019).

En la primera búsqueda se tuvo en cuenta artículos con los términos "legitim*" and "strategi*" or "action*" and "firm*" or "business" or "organization*" or "ventur*" en el tema. En la segunda búsqueda se tuvo en cuenta artículos con los términos "legitim*" and "achieve" or "reach" or "gain" or "get" or "lose" or "maintain" or "preserve" and "firm*" or "business" or "organization*" or "ventur*" en el tema. Los términos de ambas búsquedas fueron obtenidos de diversas búsquedas previas para conocer los términos más utilizados por los artículos científicos en esta materia. En ambas búsquedas se seleccionó todos los años y se refinaron los datos por: tipos de documentos (article); áreas de investigación (Business economics) y revistas (Academy of Management Journal; Organization Science; Strategic Management Journal; Journal of Management; Academy of Management Review; Journal of Business Venturing; Entrepreneurship Theory and Practice; Journal of Small Business Management; Administrative Science Quarterly; Small Business Economics; Strategic Entrepreneurship Journal). Las revistas seleccionadas seguimos las utilizadas por los autores Rawhouser et al. (2017).

De la primera búsqueda se obtuvieron 214 artículos con h-index 74 y un promedio de citas por elemento 95.5. En la segunda búsqueda se obtuvieron 47 artículo con h-index 25 y un promedio de citas por elemento 106.87. Posteriormente, en la fase de cribado, se eliminaron 21 resultados duplicados. La base de datos quedó en 240 resultados, con artículos publicados desde 1992 a junio de 2019. Continuando con la fase de cribado, se procede a leer el resumen y la introducción (en algunos casos fue necesario buscar información en otras secciones del documento) con el objetivo de asegurar que se ajustase mejor al propósito de estudio, al igual que lo hacen otros autores, por ejemplo, Urbano et al. (2019). Se excluyeron aquellos que no estaban disponibles a texto completo 
electrónicamente $(n=12)$. También se excluyeron aquellos documentos que trataban las estrategias de legitimidad sólo en el marco teórico, las limitaciones o la discusión/conclusión ( $n=40)$, al igual que los hacen otros autores, por ejemplo, Rawhouser et al. (2017). Se excluyeron aquellos que no respondían al propósito de estudio (n=79). Finalmente, fueron seleccionados 109 artículos tras los criterios de exclusión.

Posteriormente, se paso a realizar la fase de elegibilidad, tras realizar una lectura exhaustiva, se eliminaron 4 artículos; (1) por tratar de las acciones de los stakeholders (Eesley \& Lenox, 2006); (2) por tratar de por qué, cómo y en que secuencia las grandes empresas multinacionales implementan controles en sus redes de subsidiarias extranjeras (Brenner \& Ambos, 2013); (3) por tratar del modo en que el gobierno de una empresa multinacional incide en el proceso de transferencia de prácticas (Fortwengel, 2017); (4) por tratar de cómo las presiones de cambio institucional en un entorno autoritario, el activismo cívico, la burocracia gubernamental y los medios de comunicación afectan a las respuestas del gobierno a los problemas ambientales de las empresas chinas (Marquis \& Bird, 2018).

\section{Resultados}

Los 105 artículos seleccionados engloban el período de 1992 a junio de 2019. Las mayores publicaciones se han producido en el año 2014 y 2013 con 11 y 10 publicaciones, respectivamente. De los 105 artículos seleccionados, 85 tienen un carácter práctico (representa el 80,95\% del total de los artículos) y 20 son teóricos (representa el $19,05 \%$ del total de los artículos). Las dos revistas que más artículos han resultado de la selección ha sido Academy of Management Journal y Organization Science, con 26 y 22 artículos respectivamente, cada una de ellas.

El trabajo de los seleccionados con mayor número de citas es el titulado "Fools rush in? The institutional context of industry creation" (Aldrich \& Fiol, 1994) con 1.375 citas. Los cinco siguientes trabajos con mayores citas son: (1) "Customization or conformity? An institutional and network perspective on the content and consequences of TQM adoption" (Westphal et al., 1997) con 664 citaciones; (2) "Beyond survival: Achieving new venture growth by building legitimacy" (Zimmerman \& Zeitz, 2002) con 660 citaciones; (3) "Does isomorphism legitimate?" (Deephouse, 1996) con 553 citaciones; (4) "Acquiring organizational legitimacy through illegitimate actions - a marriage of institutional and impression management theories" (Elsbach \& Sutton, 1992) con 496 citaciones y; (5) "Inside the hybrid organization: selective coupling as a response to competing institutional logics" (Pache \& Santos, 2013) con 409 citaciones. Existe una media de 110,33 número de citas por artículo. Si bien, los trabajos publicados en los últimos años (2017 a junio de 2019) han recibido menos citaciones, lo que hace bajar la media de citaciones por artículo.

Los tipos de legitimidad que puede obtener una empresa realizando las diferentes acciones o estrategias que han sido obtenidas de los resultados analizados en las investigaciones científicias son: legitimidad cognitiva, legitimidad pragmática, legitimidad sociopolítica, legitimidad social, legitimidad normativa, legitimidad regulatoria, legitimidad moral, legitimidad externa e interna y legitimidad global.

La mayoría de las investigaciones se centran en estudiar las acciones y estrategias que debe seguir la empresa para lograr o mantener la legitimidad (e.g, Aldrich \& Fiol, 1994; David et al., 2013; Deephouse, 1996; Li et al., 2007; Petkova et al., 2013; van Werven et al., 2015; Wang \& Qian, 2011). Son muy pocas las investigaciones que se centran en la perdida de la legitimidad: por ejemplo, en la pérdida de legitimidad provocada por las decepciones en las expectativas esperadas por parte de los stakeholders ante una historia proyectada por la empresa (Garud et al., 2014) o cómo responden las organizaciones cuando se amenaza la legitimidad de todo un campo o sector (Desai, 2011). En la tabla 1 se pueden observar las diferentes estrategias o acciones que pueden seguir una empresa para lograr o mantener la legitimidad. Las mayores investigaciones se realizan en relación a las acciones o estrategias de gestión de impresiones y las estrategias de conformidad, diferenciación y 
manipulación del entorno por parte de las empresas. Como se puede observar en la tabla 1, las principales estrategias o acciones para obtener o mantener la legitimidad están relacionadas con: (1) la gestión de impresiones; (2) los productos y estudios de mercado; (3) las estrategias de conformidad, diferenciación y manipulación del entorno; (4) la implementación de valores sociales; (5) la gestión del personal y (6) alianzas y asociaciones con terceros.

Tabla 1

Estrategias o acciones contenidad en las investigaciones científicas

\begin{tabular}{|c|c|c|c|c|}
\hline $\begin{array}{c}\text { № } \\
\text { Artículos }\end{array}$ & $\begin{array}{l}\text { Estrategia o } \\
\text { acción } \\
\text { principal }\end{array}$ & $\begin{array}{l}\text { Estrategias o acciones } \\
\text { concretas: }\end{array}$ & $\begin{array}{l}\text { Tipo de } \\
\text { legitimidad } \\
\text { principal }\end{array}$ & Autor/es y año de publicación \\
\hline \multirow{7}{*}{48} & \multirow{7}{*}{$\begin{array}{l}\text { Gestión de } \\
\text { impresiones }\end{array}$} & Retórica (18) & \multirow{7}{*}{$\begin{array}{l}\text { Legitimidad } \\
\text { cognitiva }\end{array}$} & $\begin{array}{l}\text { Aldrich, HE; Fiol, CM (1994); Rindova, VP; Becerra, M; Contardo, I (2004); Green, Sandy Edward, Jr.; Li, } \\
\text { Yuan; Nohria, Nitin (2009); Drori, Israel; Honig, Benson; Sheaffer, Zachary (2009); Rutherford, Matthew W.; } \\
\text { Buller, Paul F.; Stebbins, J. Michael (2009); Vaara, Eero; Monin, Philippe (2010); Cornelissen, Joep P.; } \\
\text { Clarke, Jean S. (2010); Meyer, Renate E.; Hoellerer, Markus A. (2010); Desai, Vinit M. (2011); Vaara, Eero; } \\
\text { Tienari, Janne (2011); Mair, Johanna; Marti, Ignasi; Ventresca, Marc J. (2012); Garud, Raghu; Schildt, Henri } \\
\text { A.; Lant, Theresa K. (2014); Dalpiaz, Elena; Tracey, Paul; Phillips, Nelson (2014); Parhankangas, Annaleena; } \\
\text { Ehrlich, Michael (2014); Rhee, Eunice Y.; Fiss, Peer C. (2014); Harmon, Derek J.; Green, Sandy E., Jr.; } \\
\text { Goodnight, G. Thomas (2015); van Werven, Ruben; Bouwmeester, Onno; Cornelissen, Joep P. (2015); } \\
\text { Hoefer, Rolf L.; Green, Sandy E., Jr. (2016); }\end{array}$ \\
\hline & & $\begin{array}{c}\text { Medios de } \\
\text { comunicación (2) }\end{array}$ & & ELSBACH, KD; SUTTON, RI (1992); Petkova, Antoaneta P.; Rindova, Violina P.; Gupta, Anil K. (2013); \\
\hline & & $\begin{array}{l}\text { Tácticas de ofuscación } \\
\text { anticipatorias (2) }\end{array}$ & & $\begin{array}{l}\text { Elsbach, KD; Sutton, Rl; Principe, KE (1998); Kibler, Ewald; Mandl, Christoph; Kautonen, Teemu; Berger, } \\
\text { Elisabeth S. C. (2017); }\end{array}$ \\
\hline & & $\begin{array}{l}\text { Identidad organizativa } \\
\text { (8) }\end{array}$ & & $\begin{array}{l}\text { Human, SE; Provan, KG (2000); Creed, WED; Scully, MA; Austin, JR (2002); Li, Jiatao; Yang, Jing Yu; Yue, } \\
\text { Deborah R. (2007); Gioia, Dennis A.; Price, Kristin N.; Hamilton, Aimee L.; Thomas, James B. (2010); King, } \\
\text { Brayden G.; Clemens, Elisabeth S.; Fry, Melissa (2011); Fisher, Greg; Kotha, Suresh; Lahiri, Amrita (2016); } \\
\text { Hsu, Greta; Kocak, Ozgecan; Kovacs, Balzs (2018); Irwin, Jennifer; Lahneman, Brooke; Parmigiani, Anne } \\
\text { (2018); }\end{array}$ \\
\hline & & $\begin{array}{l}\text { Acciones simbólicas } \\
\text { (13) }\end{array}$ & & $\begin{array}{l}\text { Deeds, DL; Decarolis, D; Coombs, JE (1997); Fanelli, Angelo; Misangyi, Vilmos F. (2006); Zott, Christoph; } \\
\text { Huy, Quy Nguyen (2007); Lamin, Anna; Zaheer, Srilata (2012); Vergne, Jean-Philippe (2012); McDonnell, } \\
\text { Mary-Hunter; King, Brayden (2013); Helms, Wesley S.; Patterson, Karen D. W. (2014); de Vaujany, Francois- } \\
\text { Xavier; Vaast, Emmanuelle (2014); Marquis, Christopher; Qian, Cuili (2014); Canales, Rodrigo (2016); } \\
\text { Hampel, Christian E.; Tracey, Paul (2017); Goldfarb, Brent; Zavyalova, Anastasiya; Pillai, Sandeep (2018); } \\
\text { Islam, Mazhar; Fremeth, Adam; Marcus, Alfred (2018); }\end{array}$ \\
\hline & & $\begin{array}{l}\text { Gestión de emociones } \\
\text { (1) }\end{array}$ & & Huy, Quy; Zott, Christoph (2019); \\
\hline & & $\begin{array}{l}\text { Lógicas institucionales } \\
\text { (4) }\end{array}$ & & $\begin{array}{l}\text { Pache, Anne-Claire; Santos, Filipe (2013); David, Robert J.; Sine, Wesley D.; Haveman, Heather A. (2013); } \\
\text { Boone, Christophe; Oezcan, Serden (2016); Arshed, Norin; Chalmers, Dominic; Matthews, Russell (2019); }\end{array}$ \\
\hline \multirow{4}{*}{16} & \multirow{4}{*}{$\begin{array}{l}\text { Productos y } \\
\text { estudios del } \\
\text { mercado. }\end{array}$} & $\begin{array}{l}\text { Definición del } \\
\text { producto e } \\
\text { importancia de la } \\
\text { elección de la forma } \\
\text { de intercambio de } \\
\text { productos (5) }\end{array}$ & \multirow{4}{*}{$\begin{array}{l}\text { Legitimidad } \\
\text { moral }\end{array}$} & $\begin{array}{l}\text { Dougherty, D; Heller, T (1994); Choi, YR; Shepherd, DA (2005); Brush, Candida G.; Manolova, Tatiana S.; } \\
\text { Edelman, Linda F. (2008); Anteby, Michel (2010); O'Neil, Isobel; Ucbasaran, Deniz (2016); }\end{array}$ \\
\hline & & $\begin{array}{l}\text { Implementación de } \\
\text { programas de gestión } \\
\text { de calidad (4) }\end{array}$ & & $\begin{array}{l}\text { Westphal, JD; Gulati, R; Shortell, SM (1997); Payne, G. Tyge (2006); Boiral, Olivier (2007); Yeung, Andy C. L.; } \\
\text { Lo, Chris K. Y.; Cheng, T. C. E. (2011); }\end{array}$ \\
\hline & & $\begin{array}{l}\text { Desarrollo de nuevos } \\
\text { productos y cambios } \\
\text { de inversiones (5) }\end{array}$ & & $\begin{array}{l}\text { Deeds, DL; DeCarolis, D; Coombs, J (2000); Bell, R. Greg; Filatotchev, Igor; Aguilera, Ruth V. (2014); Zietsma, } \\
\text { Charlene; Lawrence, Thomas B. (2010); Bitektine, Alex; Haack, Patrick (2015); Benner, Mary J.; } \\
\text { Ranganathan, Ram (2012); }\end{array}$ \\
\hline & & $\begin{array}{l}\text { Conocimiento de los } \\
\text { clientes (2) }\end{array}$ & & Lee, K; Pennings, JM (2002); Shepherd, DA; Zacharakis, A (2003); \\
\hline \multirow{4}{*}{22} & \multirow{4}{*}{$\begin{array}{l}\text { Estrategias } \\
\text { empresariales } \\
\text { relacionadas } \\
\text { con el } \\
\text { entorno }\end{array}$} & Conformidad (10) & \multirow{4}{*}{$\begin{array}{l}\text { Legitimidad } \\
\text { normativa y } \\
\text { regulativa }\end{array}$} & $\begin{array}{l}\text { Zimmerman, MA; Zeitz, GJ (2002)*; Tornikoski, Erno T.; Newbert, Scott L. (2007); Miller, Danny; Le Breton- } \\
\text { Miller, Isabelle; Lester, Richard H. (2013); Kistruck, Geoffrey M.; Webb, Justin W.; Sutter, Christopher J.; } \\
\text { Bailey, Anastasia V. G. (2015); Smith, Edward B.; Chae, Heewon (2016); Lawrence, Thomas B. (2017); } \\
\text { Verhaal, J. Cameron; Hoskins, Jake D.; Lundmark, Leif W. (2017); Kuratko, Donald F.; Fisher, Greg; } \\
\text { Bloodgood, James M.; Hornsby, Jeffrey S. (2017); Tracey, Paul; Dalpiaz, Elena; Phillips, Nelson (2018)*; } \\
\text { Durand, Rodolphe; Hawn, Olga; loannou, loannis (2019) }\end{array}$ \\
\hline & & Diferenciación (2) & & McKnight, Brent; Zietsma, Charlene (2018); Wang, Taiyuan; Thornhill, Stewart; De Castro, Julio O. (2017); \\
\hline & & $\begin{array}{l}\text { Manipulación del } \\
\text { entorno (5) }\end{array}$ & & $\begin{array}{l}\text { Quy Nguyen Huy; Corley, Kevin G.; Kraatz, Matthew S. (2014); Lee, Chuan-Kai; Hung, Shih-Chang (2014); } \\
\text { Gurses, Kerem; Ozcan, Pinar (2015); Hunt, Richard A. (2015); Tocher, Neil; Oswald, Sharon L.; Hall, Dianne } \\
\text { J. (2015); }\end{array}$ \\
\hline & & $\begin{array}{l}\text { Isomorfismo } \\
\text { organizacional (5) }\end{array}$ & & $\begin{array}{l}\text { Deephouse, DL (1996); Volberda, Henk W.; van der Weerdt, Niels; Verwaal, Ernst; Stienstra, Marten; } \\
\text { Verdu, Antonio J. (2012); Souitaris, Vangelis; Zerbinati, Stefania; Liu, Grace (2012); Miller, Stewart Robert; } \\
\text { Indro, Daniel C.; Richards, Malika; Chng, Daniel Han Ming (2013)*; Tan, Justin; Shao, Yunfei; Li, Wan } \\
(2013)^{*} \text {; }\end{array}$ \\
\hline 8 & $\begin{array}{l}\text { Valores } \\
\text { sociales }\end{array}$ & $\begin{array}{c}\text { Filantropía } \\
\text { corporativa (5) }\end{array}$ & & $\begin{array}{l}\text { Tyler, TR; Blader, SL (2005); Chiu, Shih-Chi; Sharfman, Mark (2011); Nason, Robert S.; Bacq, Sophie; Gras, } \\
\text { David (2018); Wang, Heli; Qian, Cuili (2011); Keating, Andrew; Geiger, Susi; McLoughlin, Damien (2014); }\end{array}$ \\
\hline
\end{tabular}




\begin{tabular}{|c|c|c|c|c|}
\hline $\begin{array}{c}\text { № } \\
\text { Artículos }\end{array}$ & $\begin{array}{l}\text { Estrategia o } \\
\text { acción } \\
\text { principal }\end{array}$ & $\begin{array}{l}\text { Estrategias o acciones } \\
\text { concretas: }\end{array}$ & $\begin{array}{c}\text { Tipo de } \\
\text { legitimidad } \\
\text { principal }\end{array}$ & Autor/es y año de publicación \\
\hline & & $\begin{array}{l}\text { Responsabilidad social } \\
\text { corporativa (1) }\end{array}$ & \multirow{2}{*}{$\begin{array}{l}\text { Legitimidad } \\
\text { social o } \\
\text { Sociopolítica }\end{array}$} & Hawn, Olga; loannou, loannis (2016); \\
\hline & & $\begin{array}{l}\text { Reclamos a través de } \\
\text { activistas (2) }\end{array}$ & & Rao, H (2004); Reid, Erin M.; Toffel, Michael W. (2009); \\
\hline \multirow{3}{*}{5} & \multirow{3}{*}{$\begin{array}{l}\text { Gestión del } \\
\text { personal }\end{array}$} & Incentivos (1) & \multirow{3}{*}{$\begin{array}{l}\text { Legitimidad } \\
\text { global }\end{array}$} & Westphal, JD; Zajac, EJ (1998); \\
\hline & & $\begin{array}{l}\text { Despido de directivos } \\
\text { (1) }\end{array}$ & & Arthaud-Day, Marne L.; Certo, S. Trevis; Dalton, Catherine M.; Dalton, Dan R. (2006); \\
\hline & & $\begin{array}{l}\text { Relaciones con los } \\
\text { stakeholders (3) }\end{array}$ & & $\begin{array}{l}\text { Gibson, Cristina B.; Dibble, Rebekah (2013); Lee, Brandon H.; Hiatt, Shon R.; Lounsbury, Michael (2017); } \\
\text { Eckardt, Rory; Skaggs, Bruce C.; Lepak, David P. (2018); }\end{array}$ \\
\hline 6 & $\begin{array}{l}\text { Alianzas y } \\
\text { asociaciones } \\
\text { con otras } \\
\text { empresas }\end{array}$ & $\begin{array}{l}\text { Afiliación con terceros } \\
\qquad(6)\end{array}$ & $\begin{array}{l}\text { Legitimidad } \\
\text { global }\end{array}$ & $\begin{array}{l}\text { Rodriguez, P; Uhlenbruck, K; Eden, L (2005); Drees, Johannes M.; Heugens, Pursey P. M. A. R. (2013); } \\
\text { Khoury, Theodore A.; Junkunc, Marc; Deeds, David L. (2013); Plummer, Lawrence A.; Allison, Thomas H.; } \\
\text { Connelly, Brian L. (2016); Desai, Vinit M. (2018); Zheng, Weiting; Ni, Na; Crilly, Donal (2019); }\end{array}$ \\
\hline
\end{tabular}

*podrían estar en varias estrategias o acciones dentro de las relacionadas con el entorno

Fuente: Elaboración propia.

\subsection{Hacer uso de tácticas de gestión de impresiones}

Los empresarios pueden hacer uso de tácticas de gestión de impresiones para obtener legitimidad (principalmente legitimidad cognitiva). La gestión de impresiones se refiere a cualquier acción creada con el fin de influir en las percepciones de los stakeholders de una organización. Las acciones de gestión de impresiones pueden consistir, entre otras, en desarrollar relaciones de confianza con las partes interesadas claves, en utilizar el lenguaje y la narrativa para tratar de convencerlas o disimular una acción ilícita; en utilizar a los medios de comunicación para sensibilizar a los stakeholders o a través del desarrollo de la identidad organizativa.

\subsubsection{Retórica}

Una de las principales acciones que puede seguir una empresa para obtener o mantener la legitimidad es desarrollar relaciones de confianza con los grupos de interés a través del uso de la palabra (retórica). Es importante el uso del lenguaje para adquirir o aumentar la legitimidad de las empresas (e.g. Green et al., 2009): (1) el uso del lenguaje de guerra cuando existe una alta competitividad entre empresas atraerá más atención y apoyo de los stakeholders (Rindova et al., 2004); (2) el uso de scripts (guiones) generan legitimidad e identidad organizativa (Drori et al., 2009); (3) el uso del discurso en los procesos de fusión de empresas para obtener legitimidad (Vaara \& Monin, 2010; Vaara \& Tienari, 2011); (4) las nuevas empresas pueden utilizar analogías o metáforas para adquirir legitimidad cognitiva (Cornelissen \& Clarke, 2010); (5) Mair et al. (2012) demuestran cómo la retórica y la cultura pueden usarse como herramientas por un actor intermedio para transmitir legitimidad, mediante la capacidad de sensemaking (facilitando concienciación y conocimiento) y mediante la recombinación de normas y tradiciones; (6) Aldrich \& Fiol (1994) proponen que los fundadores de una nueva industria (nuevos mercados / nuevas formas organizativas) para obtener legitimidad cognitiva utilicen un lenguaje y un comportamiento simbólicos, fomenten la convergencia de un producto o servicio dominante, promuevan su actividad a través de actores externos y creen vínculos con los currículos educativos establecidos. Sin embargo, para obtener legitimidad sociopolítica dichos autores proponen que los socios fundadores creen historias coherentes internamente, se movilicen por la acción colectiva, negocien y se comprometan con otras industrias y realicen actividades de marketing colectivo y lobbying efforts.

El discurso también puede deslegitimar cuando estamos ante una fusión de empresas. Vaara \& Monin (2010) establecen, entre otras conclusiones, que la legitimación discursiva puede tener consecuencias no deseadas cuando el discurso mismo creo expectativas poco realistas e ilusorias; como la ocultación de los sentidos son mecanismos poderosos a través de los cuales el discurso impacta la acción organizacional y hay una variedad de estrategias generales (des) naturalización, racionalización, autorización y moralización que pueden usarse para legitimación y deslegitimación. Desai (2011) establece que, para mantener o reparar la legitimidad de todo un sector tras una crisis provocada por alguna organización perteneciente al sector, las empresas del sector deben 
aúnar sus esfuerzos de relaciones públicas para que a través de la retórica y el discurso se minimicen los efectos negativos hacia el sector.

Rutherford et al. (2009) analizan las mentiras o tergiversaciones de la verdad utilizadas por los nuevos empresarios para obtener la legitimidad. Los autores recomiendan al empresario de nuevas empresas que: (1) deben hacer todo lo posible para recopilar información adecuada y precisa (por ejemplo, producto, finanzas, etc.); (2) realizar una reflexión cuidadosa sobre las fortalezas y debilidades propias y de su empresa; (3) las tergiversaciones deliberadas de los hechos a los stakeholders deben de racionalizarlas, incluidas las que producen más beneficios que daños, ya que pueden afectar a la confianza con los stakeholders y; (4) crear un código de ética claro al inicio de la actividad.

\subsubsection{Medios de comunicación}

Otra estrategia que pueden seguir las nuevas organizaciones para obtener legitimidad es atraer la atención de los medios de comunicación para realizar actividades de sensibilización y difusión de la información y así poder llegar al mayor número de stakeholders (e.g. Petkova et al., 2013). Por ejemplo, emplear personal creíble y desviar la atención de los medios negativos hacia los fines positivos de las acciones ilegitimas (Elsbach \& Sutton, 1992).

\subsubsection{Tácticas de ofuscación anticipatorias}

Los empresarios también para evitar posibles controversias o defenderse de desafíos esperados específicos a las prácticas organizativas que va a tener efectos negativos o cuando no está claro que la organización sea responsable de un evento negativo se puede utilizar tácticas de ofuscación anticipatorias, como distraer, abrumar o disminuir la atención a un evento próximo e inducir emociones que provoquen un cumplimiento sin sentido de las solicitudes de la organización e inducir emociones en la audiencia que simplifiquen el proceso de información de los cargos (Eisbach et al., 1998). Las estrategias que deben seguir los empresarios que buscan legitimidad posterior a un fracaso con el público en general son: (1) atribuir la causa del fracaso a fuerzas externas a la empresa; (2) que los factores que causaron el fracaso no estaban bajo el control del emprendedor y; (3) que es poco probable que el fracaso vuelva a ocurrir (Kibler et al., 2017).

\subsubsection{Identidad organizativa}

Otra forma para que las empresas adquieran legitimidad es a través de la formación de una identidad organizativa (Gioia et al., 2010). Se pueden legitimar las cuentas a través de la construcción de identidades sociales (Creed et al., 2002). Li et al. (2007) tratan cómo las filiales extranjeras de propiedad absoluta ganan legitimidad en el país anfitrión, combinando identidad, comunidad y audiencia. La filial extranjera para obtener una mayor legitimación debe adoptar una identidad similar a otras filiales extranjeras establecidas en el país anfitrión que hayan sido legitimadas. Además, obtienen una mayor legitimidad si la propiedad absoluta proviene del mismo país de origen y operan en la misma industria que otras filiales extranjeras establecidas en el país anfitrión. Además, la filial extranjera debe buscar establecerse en un país anfitrión que sea receptivo y que le acepte, ya que la legitimidad depende también de la opinión pública del país anfitrión.

\subsubsection{Acciones simbólicas}

Otra estrategia que pueden seguir las nuevas organizaciones para obtener legitimidad es mediante el uso de acciones simbólicas. Zott \& Huy (2007) identifican cuatro categorías de acción simbólica que facilitan la adquisición de recursos y, por tanto, la legitimidad de una empresa: transmitir la credibilidad personal del empresario, la organización profesional, el logro de la organización y la calidad de las relaciones con los grupos

de interés. También se logra legitimidad a través del carisma de los directores ejecutivos (CEO) (Fanelli \& Misangyi, 2006). 
Las acciones simbólicas pueden consistir en la emisión o no de informes de responsabilidad social corporativa (Marquis \& Qian, 2014); en el espacio físico que ocupa una empresa (De Vaujany \& Vaast, 2014; Deeds et al., 1997); la participación en concursos, por ejemplo, la participación en una carrera de vehículos por parte de una empresa automovilística (Goldfarb et al., 2018); ganar prestigiosos premios, subvenciones o becas de investigación del gobierno pueden ayudar a pasar el umbral de legitimidad (Islam et al., 2018); en utilizar reclamos prosociales después de que se anuncie un boicot para mejorar su imagen pública y no perder su legitimidad y reputación (McDonnell \& King, 2013); en utilizar audiencias defensoras de una etiqueta o estigma negativo que tiene una empresa o un sector (Helms \& Patterson, 2014) y, por último, una acción simbólica puede consistir en utilizar asociaciones categóricas (Vergne, 2012).

\subsubsection{Gestión de emociones}

Otra forma de obtener legitimidad una empresa es a través de la gestión de emociones. Los gerentes también pueden regular sus propias emociones y las de otros grupos de interés para obtener legitimidad la empresa. En relación a las acciones que deben realizar los gerentes para regular la emoción de los stakeholders y obtener legitimidad y recursos para la empresa son: mantener un diálogo abierto sobre la oportunidad; controlar la exhibición de emociones; y mostrar consideración y apoyo de los grupos de interés clave (Q. Huy \& Zott, 2019).

\subsubsection{Lógicas institucionales}

Cómo las organizaciones hibridas, que incorporan lógicas institucionales competidoras, gestionan o manipulan estas lógicas a nivel intraorganizacional para obtener legitimidad de los stakeholders es objeto del trabajo de Pache \& Santos (2013) que demuestran "that hybrids that are persistently embedded in competing institutional logics combined elements of the competing social welfare and commercial logics by selectively coupling intact demands imposed by each logic, instead of adopting traditional strategies of decoupling or compromising" (Pache \& Santos, 2013, p.993). David et al. (2013) estudian cómo legitimar nuevos tipos de organizaciones en campos emergentes. Para ello, deben recurrir a lógicas externas a su campo, afiliarse con autoridades y élites externas y enfatizar los beneficios para la sociedad en general.

\subsection{Productos y estudios de mercado}

Existen autores que insisten en que, a través de los productos y los estudios de mercado, se puede legitimar una empresa. Este tipo de acciones están relacionados principalmente, entre otros, con la legitimidad moral. Se trata de acciones relacionadas con la importancia de definir el producto, sobretodo en empresas tecnológicas (Dougherty \& Heller, 1994), desarrollar nuevos productos (Deeds et al., 2000), la forma en que se intercambian los bienes y no solo lo que se comercializa (Anteby, 2010), ajustarse a la demanda de calidad del producto, a pesar de que suponga un decremento de la eficiencia llamado "equifinalidad óptima" (Tyge Payne, 2006), la implementación de programas de calidad (Westphal et al., 1997) o implementación de las normas ISO (Boiral, 2007) y un mayor conocimiento del mercado al que se dirige la empresa. Una nueva empresa puede realizar de estudios de mercado para lograr o fomentar su legitimidad organizativa: (1) La legitimidad de una empresa, ante una nueva forma organizativa, se puede fomentar a través de la retroalimentación del mercado. Para la retroalimentación del mercado es importante la selección de la población (Lee \& Pennings, 2002); (2) La legitimidad de una nueva empresa se puede lograr a través del conocimiento de los clientes. Por ejemplo, Shepherd \& Zacharakis (2003) establecen que una nueva empresa puede alcanzar la legitimidad cognitiva a través de un mayor conocimiento de los productos, la organización y la administración de la empresa por parte de los potenciales clientes.

Dependiendo del momento en que se encuentre la organización (al comienzo o tras unos años de antigüedad), si actúa en el mercado nacional o internacional, o la novedad del producto, deberá centrarse en desarrollar más uno de los aspectos que otros. Por ejemplo, "entrepreneurs should be able to create the assets of newness, such 
as affective congruence and strategic flexibility, during opportunity exploration, whereas they should be able to shift their focus to lowering the liabilities of newness, such as product/service reliability, as they move to the fullscale operations." (Choi \& Shepherd, 2005, p.592).

\subsection{Estrategias empresariales relacionadas con el entorno}

Se trata estrategias de conformidad, diferenciación y manipulación del entorno que están relacionadas principalmente con la legitimidad normativa y regulativa. Dependiendo de la etapa del ciclo de vida en que se encuentre una empresa, optará más por la conformidad o asimilación a otras empresas o por la diferenciación de sus competidores.

Zimmerman \& Zeitz (2002) establecen cuatro estrategias para obtener legitimidad las nuevas empresas: (1) conformidad a las normas, (2) selección de un entorno favorable para ellas, (3) manipulación del entorno mediante la realización de cambios en el entorno para lograr la coherencia entre la organización y su entorno y (4) creación del contexto social (reglas, normas, valores, creencias, modelos, etc.). También indican que las nuevas empresas pueden combinar selección y manipulación con creación y conformidad. A través de estas estrategias, la nueva empresa puede obtener cuatro tipos de legitimidad: regulativa, normativa, cognitiva e industrial. Cumplir con las normas y reglas prescritas por las instituciones otorga legitimidad a la empresa a través del registro formal de una empresa (Kistruck et al., 2015). Wang et al. (2017) demuestran que para obtener legitimidad una empresa es importante que la orientación empresarial esté enfocada a satisfacer las expectativas cognitivas, regulativas y normativas de las partes interesadas.

El isomorfismo aumenta la legitimidad organizacional (e.g. Deephouse, 1996). El isomorfismo estratégico consiste en la similitud de la estrategia de una organización con la estrategia de otras organizaciones de su industria. Souitaris et al. (2012) analizan cómo una subunidad organizacional, que opera en dos entornos institucionales diferentes, debe enfocar su isomorfismo dependiendo con quién busca legitimidad. Si busca una legitimidad interna debe enfocarse hacia "endoisomorphism". Si busca la legitimidad externamente hacia la industria debe enfocar su isomorfismo hacia "exoisomorphism".

Una empresa puede seguir una estrategia de conformidad para introducir sus nuevos productos en un nuevo mercado utilizando una denominación que se asocie un nombre conocido de productos existentes (e.g. E. B. Smith \& Chae, 2016; Verhaal et al., 2017). Durand et al. (2019) establece un modelo teórico de cuándo y cómo las organizaciones responden a las presiones normativas en busca de obtener o mantener la legitimidad. Los gerentes en función de su percepción de la importancia del problema y del costo-beneficio de la movilización de recursos para abordar el problema, van a optar por abordar el problema o no abordarle (inacción). En caso de abordar el problema pueden realizar dos acciones: cumplimiento o conformidad.

Tracey et al. (2018) estudian cómo se legitiman nuevas formas organizativas en un entorno diferente (Business incubation) como es el caso del emprendimiento tecnológico. Para alcanzar la legitimidad estas empresas deben pasar por tres fases que les llevan a seguir tres estrategias diferentes dependiendo de la fase en que se encuentre la empresa.

Sin embargo, McKnight \& Zietsma (2018) establecen la diferenciación óptima que debe tener una empresa para obtener legitimidad y éxito. Establecieron las configuraciones que logran la diferenciación equilibrada (permitieron a las empresas obtener legitimidad y, a al vez, diferenciación).

Una empresa también puede manipular el entorno para obtener legitimidad. Por ejemplo, Q. N. Huy et al. (2014) establecen cómo legitimar un proyecto emergente manipulando el contexto estratégico a través de los gerentes intermediarios para lograr legitimar el proyecto de abajo a arriba del cuadro de mandos de una empresa. 


\subsection{Legitimar a través de los valores sociales corporativos}

Las acciones o estrategias relacionadas con la implantación de valores sociales en las empresas están relacionadas principalmente con la legitimidad social o legitimidad socio-política. Que los empresarios adopten los valores sociales son esenciales con respecto a la legitimidad y al cumplimiento de las reglas por parte de los empleados. Es decir, actuar con conformidad con los códigos de conducta éticos para crear una cultura corporativa de apoyo (Tyler \& Blader, 2005). También la filantropía corporativa ayuda a las empresas a ganar legitimidad sociopolítica (Wang \& Qian, 2011). Chiu \& Sharfman (2011) muestran cómo el desempeño social corporativo ayuda a la empresa a dar respuesta a la legitimidad por parte de los stakeholders y la da una mayor visibilidad a la empresa. También realizar prácticas socialmente aceptadas permite la obtención de legitimidad a las nuevas empresas (Keating et al., 2014).

Hawn \& loannou (2016) demuestran que la acciones externas e internas de Responsabilidad Social Corporativa aumentan el valor de mercado otorgando legitimidad a la empresa. Los reclamos de activistas institucionales es fundamental en la fase temprana de obtener legitimidad para nuevas industrias (Rao, 2004). Reid \& Toffel (2009) afirman que las acciones de los accionistas y las amenazas regulatorias pueden impulsar a las empresas a adoptar prácticas coherentes con los objetivos de un movimiento social más amplio. Por ejemplo, reducir las emisiones de gases para evitar el efecto invernadero.

\subsection{Gestión del personal}

Las acciones o estrategias de gestión del personal están relacionadas principalmente con la legitimidad global. Las actividades externas que realicen los equipos de trabajo son importantes para alcanzar la legitimidad. Gibson \& Dibble (2013) indican que las actividades externas significativas que deben realizar los equipos de trabajo con los grupos de interés deben ser moderadas para que alcance su nivel más alto de efectividad.

El personal de una empresa también representa una amenaza para la legitimidad organizativa ante una crisis sociolegal o una declaración. Por ejemplo, cuando se realizan declaraciones de que se han cometido irregularidades contables. En este tipo de casos, la empresa debe despedir a los directores ejecutivos y a los directores financieros para que no se vea afectada la legitimidad de la empresa (Arthaud-Day et al., 2006).

Lee et al. (2017) establecen que para alcanzar la legitimidad una nueva categoría de mercado se requiere: reclutamiento de nuevos miembros (por ejemplo, involucrar a agricultores convencionales para lograr una agricultura orgánica); compromiso posterior (invitar a consumidores e intermediarios a participar en el marketing directo de productos orgánicos, en stands de ferias, usar anuncios locales, presentaciones del producto..) y lograr la autorización estatal en una fase temprana en el desarrollo de la categoría.

Además, la adopción de programas de incentivos a largo plazo para los administradores genera reacciones positivas en el mercado de valores, con independencia de que el plan esté implementado o no. La reacción del mercado de valores es más favorable por el uso del lenguaje de la agencia (Westphal \& Zajac, 1998).

\subsection{Crear alianzas y asociaciones con otras empresas}

Las acciones o estrategias de crear alianzas y asociaciones con otras empresas están relacionadas principalmente con la legitimidad global. Plummer et al. (2016) demuestran cómo la afiliación con terceros permite obtener legitimidad. Sobre todo, las empresas logran una mayores ganancias o acceso a los recursos si esa filiación se combina con otras señales que demuestren su madurez y compromiso, como por ejemplo, afiliación con terceros y alta experiencia gerencial o a través de las colaboraciones con stakeholders (clientes, comunidades y otros grupos). Sin embargo, se sugiere que las organizaciones no hagan uso de colaboraciones externas cuando su legitimidad esté potencialmente o ya en riesgo, bien porque se trate de stakeholders poderosos o porque las 
prácticas de la organización ya se hayan puesto en cuestión por ser inapropiadas o controvertidas (Desai, 2018). Por ejemplo, la afiliación gubernamental a las organizaciones benéficas sin animo de lucro (Zheng et al., 2019).

\section{Conclusiones}

Este estudio trata de aportar claridad y síntesis de la investigación científica sobre las acciones o estrategias para obtener, mantener o perder la legitimidad organizativa en los que se han basado los artículos publicados desde 1992 a junio de 2019, en la base de datos de Web of Science (WOS), mediante una revisión sistemática de la literatura.

Nuestro trabajo contribuye a dar una visión clara y actualizada de las publicaciones científicas sobre qué tipo de legitimidad obtienen las empresas en función de la acción o estrategia que realicen. Suchman (1995) identifica tres formas principales de legitimidad: pragmática, moral y cognitiva. Deephouse et al. (2017) indican que son cuatro tipos básicos o dimensiones de legitimidad: reguladora, pragmática, moral y cultural-cognitiva. Según nuestros resultados, debemos añadir un tipo más de legitimidad básica: la legitimidad social-sociopolítica como un tipo diferente a la legitimidad reguladora. "Sociopolitical legitimation refers to the process by which key stakeholders, the general public, key opinion leaders, or government officials accept a venture as appropriate and right, given existing norms and laws. One can measure sociopolitical legitimation by assessing public acceptance of an industry, government subsidies to the industry, or the public prestige of its leaders" (Aldrich \& Fiol, 1994, p. 648). La filantropía corporativa "it helps firms gain sociopolitical legitimacy, which enables them to elicit positive stakeholder responses and to gain political access" (Wang \& Qian, 2011, p. 1159).

Suchman (1995) estableció tres posibles desafíos de legitimidad en relación a las actividades o estrategias que podía seguir una organización: ganar, mantener y reparar la legitimidad. Deephouse et al. (2017) estableció cinco posibles escenarios de legitimación: ganar, mantener, desafiado por, responder (sustituye a defender o reparar), e institucionalmente innovador. En nuestros resultados hemos comprobado que la mayoría de los artículos científicos analizados se centran en estudiar cómo ganar legitimidad organizativa. Hay muy pocas investigaciones que se centren en analizar cómo las organizaciones mantienen la legitimidad (e.g. Q. Huy \& Zott, 2019). Por otro lado, hemos encontrado artículos que se han centrado en responder un desafío de legitimidad o reparar la legitimidad (e.g. Durand et al., 2019) y se ha observado un incremento de investigaciones relacionadas con el emprendimiento institucional innovador (e.g. David et al., 2013; C. K. Lee \& Hung, 2014).Pero, en nuestros resultados apenas hemos encontrado publicaciones científicas que analicen de forma exclusiva la pérdida de legitimidad. En nuestra opinión, a los desafíos y escenarios de legitimidad a los que se enfrenta una organización indicados por Suchman (1995) y Deephouse et al. (2017), se hace muy necesario que se investigue más sobre las causas que llevan a una organización a perder la legitimidad como un desafío o escenario más y a las acciones o estrategias que deben seguir para mantener su legitimidad ante las presiones cambiantes de los stakeholders.

Los resultados muestran las diferentes estrategias o acciones que pueden realizar las empresas para obtener o mantener la legitimidad. Se han clasificado en 6 categorías: (i) en relación con la gestión de impresiones, (ii) los productos y estudios de mercado, (iii) las estrategias empresariales de conformidad, diferenciación y manipulación del entorno, (iv) adoptar valores sociales, (v) la gestión del personal y (vi) realizar alianzas y asociaciones con otras empresas. Las estrategias o acciones para obtener o mantener legitimidad que más atención han recibido por los investigadores han sido las relacionadas con la gestión de impresiones por parte de los empresarios y las estrategias de conformidad, diferenciación y/o manipulación del entorno. En nuestros resultados cabe destacar el uso de la retórica que permite a las empresas ganar legitimidad cognitiva. A esta conclusión han llegado otros investigadores, como por ejemplo, Suddaby \& Greenwood (2005) que profundizaron sobre cómo las nuevas formas organizativas podían adquirir legitimidad cognitiva a través de la 
retórica, mediante vocabularios institucionales (la justificación del cambio debe estar relacionada con la identidad de los actores centrales) y teorizaciones del cambio (debe haber un escenario de cambio convincente).

Suchman (1995) estableció que las principales estrategias para ganar legitimidad una organización son: conformidad o adaptación al entorno (cumplir con las necesidades de la demanda, ajustarse a los ideales morales y a los modelos o estándares establecidos); seleccionar el entorno (reclutar cooperantes, definir objetivos como la eficiencia responsabilidad, confidencialidad, etc. y seleccionar etiquetas como buscar certificación explicita) y manipular el entorno (a través de la publicidad del producto, tener un historial de éxito técnico o promover la comprensibilidad y alentar al isomorfismo). En nuestro trabajo en relación a las estrategias relacionadas con el entorno, a diferencia de Suchman (1995), se determinaron que además de las estrategia de conformidad y manipulación del entorno, existían estrategias de diferenciación y de isomorfismo organizacional como dos estrategias más e independientes. Si bien en el trabajo de dicho autor nombra el isomorfismo, pero lo engloba como parte de la manipulación del entorno y, la diferenciación podría estar recogida en la selección entre entornos al tratar de diferenciarse la organización de otras empresas.

En nuestro trabajo se establecen estrategias especificas y aparecen estrategias o acciones no establecidas en el trabajo de Suchman (1995), como por ejemplo, las estrategias relacionadas con la legitimidad social o sociopolítica como la filantropía corporativa y la responsabilidad social corporativa o las estrategias de alianzas y asociaciones con otras empresas. Por otra parte, en nuestros resultados las estrategias de definir el producto e importancia de la elección de la forma de intercambio de productos, implementación de programas de calidad, desarrollo de nuevos productos, cambios de inversiones y conocimiento de los clientes que podrían englobarse como estudios de mercado, en lo que Suchman (1995) define como percibir los cambios futuros, no sólo son estrategias para mantener la legitimidad, sino también para ganar legitimidad la empresa.

Nuestro trabajo contribuye a ayudar a las empresas para que desarrollen mejores estrategias y acciones de manera proactiva para obtener o mantener la legitimidad otorgada por los stakeholders. También contribuye a que los académicos puedan tener una visión general en relación al tema tratado en dicho trabajo. Por último, se contribuye, tanto en el ámbito de investigación como académico, haciendo un estudio amplio y pormenorizado de las diferentes acciones y estrategias que pueden realizar las empresas relacionándolas con los principales tipos de legitimidad que se obtienen de cada una de ellas.

\subsection{Limitaciones y futuras investigaciones}

Este estudio tiene las limitaciones propias de una revisión literaria como la selección de las palabras claves y el uso de parámetros tomados por los investigadores, creando un cierto grado de subjetividad a las interpretaciones realizadas por los mismos. Otra limitación es el sesgo en los estudios seleccionados que puede afectar a los resultados (Whittemore et al., 2014). Por último, cabe destacar que en esta investigación se ha utilizado de manera exclusiva una base de datos obtenida de Web of Science (WOS), sin tener en cuenta otros recursos como la base de datos de Scopus donde el número de trabajos publicados es mayor. Los investigadores podrían replicar esta investigación utilizando la base de datos Scopus o comparar lo que se obtiene en WOS con SCOPUS, sin filtrar por tipo de artículos ni las revistas seleccionadas en el presente trabajo.

En el presente trabajo no hemos revisado los métodos de investigación utilizados por los artículos científicos empíricos estudiados en relación a la legitimidad organizativa. Futuras investigaciones podrían analizarlos para conocer cuáles son los métodos más apropiados o cuáles son los más utilizados en el ámbito científico de este campo.

Se precisa de un análisis del poder que tienen los stakeholders en cada una de las estrategias analizadas y ver cómo influye ese poder en las estrategias. Para ello, se requerirá previamente clasificar a los stakeholders en 
función de si tienen un poder alto en la estrategia o no; si tienen un interés alto o no; si tienen una alta influencia o no en la estrategia, etc.

También sería interesante analizar estas estrategias o acciones para obtener, mantener o perder la legitimidad distinguiendo por el tipo de organización. Por ejemplo, si se trata de nuevas empresas o no. Ya que una nueva empresa debe enfocarse a obtener la legitimidad y, por tanto, las acciones que debe realizar son diferentes a una empresa establecida en el mercado, que ya tiene adquirida la legitimidad, y sus acciones deben enfocarse a mantener esa legitimidad otorgada por los stakeholders. Otro ejemplo, sería por el tipo de sector en que opera la empresa. Ya que no serán las mismas acciones o estrategias que debe realizar una empresa que opera en una industria de alta tecnología que una organización que opera en un sector turístico.

Por último, es necesario realizar más investigaciones con el objetivo de conocer más acciones o estrategias que las empresas puedan realizar ante el desafío de mantener la legitimidad o la pérdida de legitimidad.

\section{Referencias bibliográficas}

Aldrich, H. E., \& Fiol, C. M. (1994). Fools rush in? The institutional context of industry creation. Academy of Management Review, 19(4), 645-670. Recuperado de: https://doi.org/10.2307/258740

Anteby, M. (2010). Markets, morals, and ractices of trade: Jurisdictional disputes in the U.S. commerce in cadavers. Administrative Science Quarterly, 55(4), 606-638. Recuperado de: https://doi.org/10.2189/asqu.2010.55.4.606

Archibald, M. E. (2004). Between isomorphism and market partitioning: how organizational competencies and resources foster cultural and sociopolitical legitimacy, and promote organizational survival. In Research in the Sociology of Organizations (Vol. 22). Recuperado de: https://doi.org/10.1016/S0733-558X(04)22006-7

Arthaud-Day, M. L., Certo, S. T., Dalton, C. M., \& Dalton, D. R. (2006). A changing of the guard: Executive and director turnover following corporate financial restatements. Academy of Management Journal, 49(6), 1119-1136. Recuperado de: https://doi.org/10.5465/AMJ.2006.23478165

Boiral, O. (2007). Corporate greening through ISO 14001: A rational myth? Organization Science, 18(1), 127146. Recuperado de: https://doi.org/10.1287/orsc.1060.0224

Brenner, B., \& Ambos, B. (2013). A question of legitimacy? A dynamic perspective on multinational firm control. Organization Science, 24(3), 773-795. Recuperado de: https://doi.org/10.1287/orsc.1120.0760

Chiu, S. C., \& Sharfman, M. (2011). Legitimacy, visibility, and the antecedents of corporate social performance: An investigation of the instrumental perspective. Journal of Management, 37(6), 1558-1585. Recuperado de: https://doi.org/10.1177/0149206309347958

Choi, Y. R., \& Shepherd, D. A. (2005). Stakeholder perceptions of age and other dimensions of newness. Journal of Management, 31(4), 573-596. Recuperado de: https://doi.org/10.1177/0149206304272294

Cook, D. J., Mulrow, C. D., \& Haynes, R. B. (1997). Systematic reviews: Synthesis of best evidence for clinical decisions. Annals of Internal Medicine, 126(5), 376-380. Recuperado de: https://doi.org/10.7326/00034819-126-5-199703010-00006

Cornelissen, J., \& Clarke, J. (2010). Imagining and rationalizing opportunities: Inductive reasoning and the creation and justification of new ventures. Academy of Management Review, 35(4), 539-557. Recuperado de: https://doi.org/10.5465/AMR.2010.53502700 
Creed, W. E. D., Scully, M. A., \& Austin, J. R. (2002). Clothes make the person? The tailoring of legitimating accounts and the social construction of identity. Organization Science, 13(5), 475-496. Recuperado de: https://doi.org/10.1287/orsc.13.5.475.7814

Crowther, M., Lim, W., \& Crowther, M. A. (2010). Systematic review and meta-analysis methodology. Blood, 116(17), 3140-3146. Recuperado de: https://doi.org/10.1182/blood-2010-05-280883

David, R. J., Sine, W. D., \& Haveman, H. A. (2013). Seizing opportunity in emerging fields: How institutional entrepreneurs legitimated the professional form of management consulting. Organization Science, 24(2), 356-377. Recuperado de: https://doi.org/10.1287/orsc.1120.0745

De Vaujany, F. X., \& Vaast, E. (2014). If these walls could talk: The mutual construction of organizational space and legitimacy. Organization Science, 25(3), 713-731. Recuperado de: https://doi.org/10.1287/orsc.2013.0858

Deeds, D. L., Decarolis, D., \& Coombs, J. (2000). Dynamic capabilities and new product development in high technology ventures: An empirical analysis of new biotechnology firms. Journal of Business Venturing, 15(3), 211-229. Recuperado de: https://doi.org/10.1016/S0883-9026(98)00013-5

Deeds, D. L., Decarolis, D., \& Coombs, J. E. (1997). The impact of firm-specific capabilities on the amount of capital raised in an initial public offering: Evidence from the biotechnology industry. Journal of Business Venturing, 12(1), 31-46. Recuperado de: https://doi.org/10.1016/S0883-9026(97)84970-1

Deephouse, D. L. (1996). Does isomorphism legitimate? Academy of Management Journal, 39(4), 1024-1039. Recuperado de: https://doi.org/10.2307/256722

Deephouse, D. L., Bundy, J., Tost, P. L., \& Suchman, M. C. (2017). Organizational Legitimacy: Six Key Questions. In The SAGE Handbook or Organizational Institutionalism (pp. 28-54). Vienna, Austria: SAGE

Deephouse, D. L., \& Suchman, M. (2008). Legitimacy in Organizational Institutionalism. In The SAGE Handbook of Organizational Institutionalism (pp. 49-77). Vienna, Austria: SAGE

Delmar, F., \& Shane, S. (2004). Legitimating first: Organizing activities and the survival of new ventures. Journal of Business Venturing, 19(3), 385-410. Recuperado de: https://doi.org/10.1016/S0883-9026(03)00037-5

Desai, V. M. (2011). Mass media and massive failures: Determining organizational efforts to defend field legitimacy following crises. Academy of Management Journal, 54(2), 263-278. Recuperado de: https://doi.org/10.5465/AMJ.2011.60263082

Desai, V. M. (2018). Collaborative stakeholder engagement: An integration between theories of organizational legitimacy and learning. Academy of Management Journal, 61(1), 220-244. Recuperado de: https://doi.org/10.5465/amj.2016.0315

Díez-de-Castro, E., Peris-Ortiz, M., \& Díez-Martín, F. (2018). Criteria for evaluating the organizational legitimacy: A typology for legitimacy jungle. In Organizational Legitimacy: Challenges and Opportunities for Businesses and Institutions. Switzerland: Springer. Recuperado de: https://doi.org/10.1007/978-3-319-75990-6_1

Dougherty, D., \& Heller, T. (1994). The Illegitimacy of Successful Product Innovation in Established Firms. Organization Science, 5(2), 200-218. Recuperado de: https://doi.org/10.1287/orsc.5.2.200

Drori, I., Honig, B., \& Sheaffer, Z. (2009). The life cycle of an internet firm: Scripts, legitimacy, and identity. Entrepreneurship Theory and Practice, 33(3), 715-738. Recuperado de: https://doi.org/10.1111/j.15406520.2009.00323.x 
Durand, R., Hawn, O., \& loannou, I. (2019). Willing and able: A general model of organizational responses to normative pressures. Academy of Management Review, 44(2), 299-320. Recuperado de: https://doi.org/10.5465/amr.2016.0107

Eesley, C., \& Lenox, M. J. (2006). Firm responses to secondary stakeholder action. Strategic Management Journal, 27(8), 765-781. Recuperado de: https://doi.org/10.1002/smj.536

Eisbach, K. D., Sutton, R. I., \& Principe, K. E. (1998). Averting Expected Challenges Through Anticipatory Impression Management: A Study of Hospital Billing. Organization Science, 9(1), 68-86. Recuperado de: https://doi.org/10.1287/orsc.9.1.68

Elsbach, K. D., \& Sutton, R. I. (1992). Acquiring Organizational Legitimacy Through Illegitimate Actions: A Marriage of Institutional and Impression Management Theories. Academy of Management Journal, 35(4), 699-738. Recuperado de: https://doi.org/10.5465/256313

Fanelli, A., \& Misangyi, V. F. (2006). Bringing out charisma: CEO charisma and external stakeholders. Academy of Management Review, 31(4), 1049-1061. Recuperado de: https://doi.org/10.5465/AMR.2006.22528170

Fisher, G., Kotha, S., \& Lahiri, A. (2016). Changing with the times: An integrated view of identity, legitimacy, and new venture life cycles. Academy of Management Review, 41(3), 383-409. Recuperado de: https://doi.org/10.5465/amr.2013.0496

Fortwengel, J. (2017). Practice transfer in organizations: The role of governance mode for internal and external fit. Organization Science, 28(4), 690-710. Recuperado de: https://doi.org/10.1287/orsc.2017.1135

Garud, R., Schildt, H. A., \& Lant, T. K. (2014). Entrepreneurial storytelling, future expectations, and the paradox of legitimacy. Organization Science, 25(5), 1479-1492. Recuperado de: https://doi.org/10.1287/orsc.2014.0915

Gibson, C. B., \& Dibble, R. (2013). Excess may do harm: Investigating the effect of team external environment on external activities in teams. Organization Science, 24(3), 697-715. Recuperado de: https://doi.org/10.1287/orsc.1120.0766

Gioia, D. A., Price, K. N., Hamilton, A. L., \& Thomas, J. B. (2010). Forging an identity: An insider-outsider study of processes involved in the formation of organizational identity. Administrative Science Quarterly, 55(1), 146. Recuperado de: https://doi.org/10.2189/asqu.2010.55.1.1

Goldfarb, B., Zavyalova, A., \& Pillai, S. (2018). Did victories in certification contests affect the survival of organizations in the American automobile industry during 1895-1912? A replication study. Strategic Management Journal, 39(8), 2335-2361. Recuperado de: https://doi.org/10.1002/smj.2911

Green, S. E., Li, Y., \& Nohria, N. (2009). Suspended in self-spun webs of significance: A rhetorical model of institutionalization and institutionally embedded agency. Academy of Management Journal, 52(1), 11-36. Recuperado de: https://doi.org/10.5465/AMJ.2009.36461725

Hawn, O., \& loannou, I. (2016). Mind the gap: The interplay between external and internal actions in the case of corporate social responsibility. Strategic Management Journal, 37(13), 2569-2588. Recuperado de: https://doi.org/10.1002/smj.2464

Helms, W. S., \& Patterson, K. D. W. (2014). Eliciting acceptance for "illicit" organizations: The positive implications of stigma for MMA organizations. Academy of Management Journal, 57(5), 1453-1484. Recuperado de: https://doi.org/10.5465/amj.2012.0088 
Huy, Q. N., Corley, K. G., \& Kraatz, M. S. (2014). From support to mutiny: Shifting legitimacy judgments and emotional reactions impacting the implementation of radical change. Academy of Management Journal, 57(6), 1650-1680. Recuperado de: https://doi.org/10.5465/amj.2012.0074

Huy, Q., \& Zott, C. (2019). Exploring the affective underpinnings of dynamic managerial capabilities: How managers' emotion regulation behaviors mobilize resources for their firms. Strategic Management Journal, 40(1), 28-54. Recuperado de: https://doi.org/10.1002/smj.2971

Islam, M., Fremeth, A., \& Marcus, A. (2018). Signaling by early stage startups: US government research grants and venture capital funding. Journal of Business Venturing, 33(1), 35-51. Recuperado de:

https://doi.org/10.1016/j.jbusvent.2017.10.001

Keating, A., Geiger, S., \& Mcloughlin, D. (2014). Riding the practice waves: Social resourcing practices during new venture development. Entrepreneurship Theory and Practice, 38(5), 1207-1235. Recuperado de: https://doi.org/10.1111/etap.12038

Kibler, E., Mandl, C., Kautonen, T., \& Berger, E. S. C. (2017). Attributes of legitimate venture failure impressions. Journal of Business Venturing, 32(2), 145-161. Recuperado de: https://doi.org/10.1016/j.jbusvent.2017.01.003

Kistruck, G. M., Webb, J. W., Sutter, C. J., \& Bailey, A. V. G. (2015). The double-edged sword of legitimacy in base-of-the-pyramid markets. Journal of Business Venturing, 30(3), 436-451. Recuperado de: https://doi.org/10.1016/j.jbusvent.2014.06.004

Knopf, J. W. (2006). Doing a literature review. PS - Political Science and Politics, 39(1), 127-132. Recuperado de: https://doi.org/10.1017/S1049096506060264

Lee, B. H., Hiatt, S. R., \& Lounsbury, M. (2017). Market mediators and the trade-offs of legitimacy-seeking behaviors in a nascent category. Organization Science, 28(3), 447-470. Recuperado de: https://doi.org/10.1287/orsc.2017.1126

Lee, C. K., \& Hung, S. C. (2014). Institutional entrepreneurship in the informal economy: China's shan-zhai mobile phones. Strategic Entrepreneurship Journal, 8(1), 16-36. Recuperado de: https://doi.org/10.1002/sej.1174

Lee, K., \& Pennings, J. M. (2002). Mimicry and the market: Adoption of a new organizational form. Academy of Management Journal, 45(1), 144-162. Recuperado de: https://doi.org/10.2307/3069289

Li, J., Yang, J. Y., \& Yue, D. R. (2007). Identity, community, and audience: How wholly owned foreign subsidiaries gain legitimacy in China. Academy of Management Journal, 50(1), 175-190. Recuperado de: https://doi.org/10.5465/AMJ.2007.24162209

Mair, J., Martí, I., \& Ventresca, M. J. (2012). Building inclusive markets in rural Bangladesh: How intermediaries work institutional voids. Academy of Management Journal, 55(4), 819-850. Recuperado de: https://doi.org/10.5465/amj.2010.0627

Marquis, C., \& Bird, Y. (2018). The paradox of responsive authoritarianism: How civic activism spurs environmental penalties in China. Organization Science, 29(5), 948-968. Recuperado de: https://doi.org/10.1287/orsc.2018.1212

Marquis, C., \& Qian, C. (2014). Corporate social responsibility reporting in China: Symbol or substance? Organization Science, 25(1), 127-148. Recuperado de: https://doi.org/10.1287/orsc.2013.0837 
McDonnell, M. H., \& King, B. (2013). Keeping up Appearances: Reputational Threat and Impression Management after Social Movement Boycotts. Administrative Science Quarterly, 58(3), 387-419. Recuperado de: https://doi.org/10.1177/0001839213500032

McKnight, B., \& Zietsma, C. (2018). Finding the threshold: A configurational approach to optimal distinctiveness. Journal of Business Venturing, 33(4), 493-512. Recuperado de: https://doi.org/10.1016/j.jbusvent.2018.03.004

Meyer, J. W., \& Rowan, B. (1977). Institutionalized Organizations: Formal Structure as Myth and Ceremony. American Journal of Sociology, 83(2), 340-363. Recuperado de: https://doi.org/10.1086/226550

Moher, D., Liberati, A., Tetzlaff, J., Altman, D. G., Altman, D., Antes, G., Atkins, D., Barbour, V., Barrowman, N., Berlin, J. A., Clark, J., Clarke, M., Cook, D., D’Amico, R., Deeks, J. J., Devereaux, P. J., Dickersin, K., Egger, M., Ernst, E., ... Tugwell, P. (2009). Preferred reporting items for systematic reviews and meta-analyses: The PRISMA statement. PLoS Medicine, 6(7), e1000097. Recuperado de: https://doi.org/10.1371/journal.pmed.1000097

Nagy, B., Rutherford, M., Truong, Y., \& Pollack, J. (2017). Development of the legitimacy threshold scale. Journal of Small Business Strategy, 27(3), 50-58. Recuperado de: http://libjournals.mtsu.edu/index.php/jsbs/article/view/763

Oliver, C. (1991). Strategic Responses To Processes Institutional. Academy of Management Review, 16(1), 145179. Recuperado de: https://doi.org/10.5465/AMR.1991.4279002

Pache, A. C., \& Santos, F. (2013). Inside the hybrid organization: Selective coupling as a response to competing institutional logics. Academy of Management Journal, 56(4), 972-1001. Recuperado de: https://doi.org/10.5465/amj.2011.0405

Petkova, A. P., Rindova, V. P., \& Gupta, A. K. (2013). No news is bad news: Sensegiving activities, media attention, and venture capital funding of new technology organizations. Organization Science, 24(3), 865888. Recuperado de: https://doi.org/10.1287/orsc.1120.0759

Plummer, L. A., Allison, T. H., \& Connelly, B. L. (2016). Better together? signaling interactions in new venture pursuit of initial external capital. Academy of Management Journal, 59(5), 1585-1604. Recuperado de: https://doi.org/10.5465/amj.2013.0100

Rao, H. (2004). Institutional activism in the early American automobile industry. Journal of Business Venturing, 19(3), 359-384. Recuperado de: https://doi.org/10.1016/S0883-9026(03)00036-3

Rawhouser, H., Villanueva, J., \& Newbert, S. L. (2017). Strategies and Tools for Entrepreneurial Resource Access: A Cross-disciplinary Review and Typology. International Journal of Management Reviews, 19(4), 473-491. Recuperado de: https://doi.org/10.1111/ijmr.12105

Reid, E. M., \& Toffel, M. W. (2009). Responding to public and private politics: Corporate disclosure of climate change strategies. Strategic Management Journal, 30(11), 1157-1178. Recuperado de: https://doi.org/10.1002/smj.796

Rindova, V. P., Becerra, M., \& Contardo, I. (2004). Enacting competitive wars: Competitive activity, language games, and market consequences. Academy of Management Review, 29(4), 670-686. Recuperado de: https://doi.org/10.5465/AMR.2004.14497655 
Rutherford, M. W., Buller, P. F., \& Stebbins, J. M. (2009). Ethical considerations of the legitimacy lie. Entrepreneurship Theory and Practice, 33(4), 949-964. Recuperado de: https://doi.org/10.1111/j.15406520.2009.00310.x

Shepherd, D. A., \& Zacharakis, A. (2003). A New Venture's Cognitive Legitimacy: An Assessment by Customers. Journal of Small Business Management, 41(2), 148-167. Recuperado de: https://doi.org/10.1111/1540$627 x .00073$

Smith, E. B., \& Chae, H. (2016). We do what we must, and call it by the best names: Can deliberate names offset the consequences of organizational atypicality? Strategic Management Journal, 37(6), 1021-1033. Recuperado de: https://doi.org/10.1002/smj.2386

Smith, V., Devane, D., Begley, C. M., \& Clarke, M. (2011). Methodology in conducting a systematic review of systematic reviews of healthcare interventions. BMC Medical Research Methodology, 11(15). Recuperado de: https://doi.org/10.1186/1471-2288-11-15

Souitaris, V., Zerbinati, S., \& Liu, G. (2012). Which iron cage? Endo- and exoisomorphism in corporate venture capital programs. Academy of Management Journal, 55(2), 477-505. Recuperado de: https://doi.org/10.5465/amj.2009.0709

Suchman, M. C. (1995). Managing legitimacy: strategic and institutional approaches. The Academy of Management Review, 20(3), 571-610. Recuperado de: https://doi.org/10.2307/258788

Suddaby, R., \& Greenwood, R. (2005). Rhetorical strategies of legitimacy. Administrative Science Quarterly, 50(1), 35-67. Recuperado de: https://doi.org/10.2189/asqu.2005.50.1.35

Tracey, P., Dalpiaz, E., \& Phillips, N. (2018). Fish out of water: Translation, legitimation, and new venture creation. Academy of Management Journal, 61(5), 1627-1666. Recuperado de: https://doi.org/10.5465/amj.2015.0264

Tyge Payne, G. (2006). Examining configurations and firm performance in a suboptimal equifinality context. Organization Science, 17(6), 756-770. Recuperado de: https://doi.org/10.1287/orsc.1060.0203

Tyler, T. R., \& Blader, S. L. (2005). Can businesses effectively regulate employee conduct? The antecedents of rule following in work settings. Academy of Management Journal, 48(6), 1143-1158. Recuperado de: https://doi.org/10.5465/AMJ.2005.19573114

Urbano, D., Aparicio, S., \& Audretsch, D. B. (2019). Institutional Antecedents of Entrepreneurship and Its Consequences on Economic Growth: A Systematic Literature Analysis. In International Studies in Entrepreneurship (pp. 15-56). Recuperado de: https://doi.org/10.1007/978-3-030-13373-3_2

Vaara, E., \& Monin, P. (2010). A recursive perspective on discursive legitimation and organizational action in mergers and acquisitions. Organization Science, 21(1), 3-22. Recuperado de: https://doi.org/10.1287/orsc.1080.0394

Vaara, E., \& Tienari, J. (2011). On the narrative construction of multinational corporations: An antenarrative analysis of legitimation and resistance in a cross-border merger. Organization Science, 22(2), 370-390. Recuperado de: https://doi.org/10.1287/orsc.1100.0593

van Werven, R., Bouwmeester, O., \& Cornelissen, J. P. (2015). The power of arguments: How entrepreneurs convince stakeholders of the legitimate distinctiveness of their ventures. Journal of Business Venturing. Recuperado de: https://doi.org/10.1016/j.jbusvent.2014.08.001 
Vergne, J. P. (2012). Stigmatized categories and public disapproval of organizations: A mixed-methods study of the global arms industry, 1996-2007. Academy of Management Journal, 55(5), 1027-1052. Recuperado de: https://doi.org/10.5465/amj.2010.0599

Verhaal, J. C., Hoskins, J. D., \& Lundmark, L. W. (2017). Little Fish in a Big Pond: Legitimacy Transfer, Authenticity, and Factors of Peripheral Firm Entry and Growth in the Market Center. Strategic Management Journal, 38(12), 2532-2552. Recuperado de: https://doi.org/10.1002/smj.2681

Wang, H., \& Qian, C. (2011). Corporate philanthropy and corporate financial performance: The roles of stakeholder response and political access. Academy of Management Journal, 54(6), 1159-1181. Recuperado de: https://doi.org/10.5465/amj.2009.0548

Wang, T., Thornhill, S., \& De Castro, J. O. (2017). Entrepreneurial Orientation, Legitimation, and New Venture Performance. Strategic Entrepreneurship Journal, 11(4), 373-392. Recuperado de: https://doi.org/10.1002/sej.1246

Westphal, J. D., Gulati, R., \& Shortell, S. M. (1997). Customization or conformity? An institutional and network perspective on the content and consequences of TQM adoption. Administrative Science Quarterly, 42(2), 366-394. Recuperado de: https://doi.org/10.2307/2393924

Westphal, J. D., \& Zajac, E. J. (1998). The symbolic management of stockholders: Corporate governance reforms and shareholder reactions. Administrative Science Quarterly, 43(1), 127-153. Recuperado de: https://doi.org/10.2307/2393593

Whittemore, R., Chao, A., Jang, M., Minges, K. E., \& Park, C. (2014). Methods for knowledge synthesis: An overview. Heart \& Lung: Journal of Acute and Critical Care, 43(5), 453-461. Recuperado de: https://doi.org/10.1016/j.hrtlng.2014.05.014

Zheng, W., Ni, N., \& Crilly, D. (2019). Non-profit organizations as a nexus between government and business: Evidence from Chinese charities. Strategic Management Journal, 40(4), 658-684. Recuperado de: https://doi.org/10.1002/smj.2958

Zimmerman, M. A., \& Zeitz, G. J. (2002). Beyond survival: Achieving new venture growth by building legitimacy. The Academy of Management Review, 27(3), 414-431. Recuperado de: https://www.jstor.org/stable/4134387

Zott, C., \& Huy, Q. N. (2007). How Entrepreneurs Use Symbolic Management to Acquire Resources. Administrative Science Quarterly, 52(1), 70-105. Recuperado de: https://doi.org/10.2189/asqu.52.1.70

Esta obra está bajo una Licencia Creative Commons Attribución-NoCommercial 4.0 International

(c) EY-NO 